Full Length Article

\title{
Photoluminescence of CdZnTe thick films obtained by close-spaced vacuum sublimation
}

\author{
V. Kosyak ${ }^{\mathrm{a}, \mathrm{b}}$, Y. Znamenshchykov ${ }^{\mathrm{a}}$, Aurimas Čerškus ${ }^{\mathrm{c}}$, L. Grase ${ }^{\mathrm{b}}$, Yu.P. Gnatenko ${ }^{\mathrm{d}}$, \\ A. Medvids ${ }^{b}$, A. Opanasyuk ${ }^{a}$, G. Mezinskis $^{b}$ \\ a Sumy State University, Rymskogo-Korsakova Str. 2, 4007 Sumy, Ukraine \\ ${ }^{\mathrm{b}}$ Riga Technical University, 3 Paula Valdena Str., LV-1048 Riga, Latvia

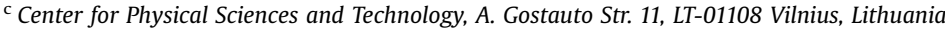 \\ ${ }^{\mathrm{d}}$ Institute of Physics of National Academy of Sciences of Ukraine, 03028 Kyiv, Ukraine
}

\section{A R T I C L E I N F O}

\section{Article history:}

Received 16 September 2015

Accepted 20 November 2015

\section{Keywords:}

II-VI crystals

Low-temperature photoluminescence

Semiconductor thick films

Residual impurity states

Point and extended defects

Optical quality

\begin{abstract}
A B S T R A C T
Polycrystalline $\mathrm{Cd}_{1-x} \mathrm{Zn}_{x} \mathrm{Te}$ thick films with thicknesses of about $30 \mu \mathrm{m}$ have been deposited on a Mo coated glass substrate by means of close-spaced vacuum sublimation technique. X-ray diffraction measurements have shown that the films obtained have only cubic zinc blende phase. The influence of $\mathrm{Zn}$ concentration on the photoluminescence (PL) spectra of $\mathrm{Cd}_{1-x} \mathrm{Zn}_{x} \mathrm{Te}$ films was investigated. This let us determine the nature and energy structure of the intrinsic defects and residual impurities in the films. The presence of the most intense acceptor bound exciton $\mathrm{A}^{\circ} \mathrm{X}$-line for $x=0.10$ and the lines of localized excitons $(x=0.32-0.44)$ in PL spectra of $\mathrm{Cd}_{1-x} \mathrm{Zn}_{x} \mathrm{Te}$ films indicates their fairly good optical quality as well as the p-type conductivity. There were also other intensive broad PL bands, caused by the recombination of donor-acceptor pairs involving complex acceptor centers, extended defects of dislocation type, and microstress in the films. It was also established a correlation between the broadening of exciton lines and the values of microstress in $\mathrm{Cd}_{1-x} \mathrm{Zn}_{x} \mathrm{Te}$ thick films. Taking into account the energy position of exciton lines, the concentration dependence of the band gap for the $\mathrm{Cd}_{1-x} Z \mathrm{n}_{x} \mathrm{Te}$ thick films is presented. (c) 2015 Elsevier B.V. All rights reserved.
\end{abstract}

\section{Introduction}

At present $\mathrm{Cd}_{1-x} \mathrm{Zn}_{x}$ Te solid solutions with tunable band gap in the spectral region between $1.50 \mathrm{eV}$ to $2.30 \mathrm{eV}$ at room temperature (for $x=0$ and $x=1.0$, respectively) are being intensively studied mainly because they are considered as suitable material for non-cooled X- and gamma rays detectors [1-4]. The presence of zinc atoms substantially increases the resistivity of these solid solutions and hence their detector performance in comparison with the CdTe crystals [5]. At the same time, crystalline defects, such as dislocations and point defects limit the performance of these materials.

In order to use these materials in micro- and optoelectronics effectively it is necessary to obtain the crystals of fairly good optical quality, which would improve their electronic properties. It should be noted that $\mathrm{Cd}_{1-x} \mathrm{Zn}_{x}$ Te crystal for $x \leq 0.30$ has a more covalent bonding and higher stacking fault energy compared to CdTe crystal, which reduces the dislocation density and twin formation [5]. This result is also supported by the analysis of XRD study, which indicates that the $\mathrm{Zn}$-Te bond is stretched by doping

\footnotetext{
E-mail address: yuriygnatenko@ukr.net (Yu.P. Gnatenko).
}

with $\mathrm{Zn}$, but the $\mathrm{Cd}-\mathrm{Te}$ bond is compressed. Thus, the $\mathrm{Cd}-\mathrm{Te}$ is stronger and the bond-length in $\mathrm{Cd}_{1-x} \mathrm{Zn}_{x} \mathrm{Te}$ is shorter than that of pure CdTe, which should also be responsible for the low density of dislocation and twin formation in $\mathrm{Cd}_{1-x} \mathrm{Zn}_{x} \mathrm{Te}$ [6]. Besides the extended defects, deposited $\mathrm{Cd}_{1-x} \mathrm{Zn}_{x}$ Te films include intrinsic defects, which are randomly distributed in the films as well as residual impurities. These defects have strong effect on the optical and photoelectrical properties of the films and thus considerably impact on their application efficiency. At the same time, since $\mathrm{Zn}$ atoms occupy the position of $\mathrm{Cd}$ atoms and their atomic radii have different sizes, the local atomic structure of $\mathrm{Cd}_{1-x} \mathrm{Zn}_{x} \mathrm{Te}$ crystals is distorted. Furthermore, inhomogeneous distribution of $\mathrm{Zn}$ atoms also causes a distortion of the crystal lattice.

In order to obtain low cost and large-area uniform $\mathrm{Cd}_{1-x} \mathrm{Zn}_{x} \mathrm{Te}$ for X-ray imaging detectors [7] it is necessary to use films with the thickness of $30 \mu \mathrm{m}[8,9]$ and higher instead of bulk crystals. Furthermore, since $\mathrm{Cd}_{1-x} \mathrm{Zn}_{x}$ Te have p-type conductivity, they may be used as alternative top adsorber layers in solar cells with the optimal band gap about of (1.7-1.8) eV at $x \approx 0.30$ for effective conversion of light absorption into electrical power.

At present different methods of $\mathrm{Cd}_{1-x} \mathrm{Zn}_{x} \mathrm{Te}$ films deposition such as metalorganic vapor-phase epitaxy [9] metal-organic chemical vapor deposition [10], pulsed laser deposition [11], thermal 
evaporation [12,13], brush plating technique [14], magnetron sputtering [15], hot wall epitaxy [16], laser ablation [11] were used. Earlier [17], it was shown that a low cost close-spaced vacuum sublimation (CSVS) method makes it possible to deposit the stoichiometric films of II-VI semiconductors with the controllable physical properties using a glass as substrate. However, until now, the defect structure of $\mathrm{Cd}_{1-x} \mathrm{Zn}_{x}$ Te polycrystalline films deposited using this method has not been comprehensively studied.

Low temperature photoluminescence (PL) measurement is a very effective method for studying the defect's structure of various semiconductor polycrystalline films and their band structure [11,17-20]. At present, the PL spectra of bulk semiconductor crystals are studied well enough [21-24], but thin and thick films of these materials have not been thoroughly investigated.

It was found theoretically that the optimal band gap about of $2.0 \mathrm{eV}(x=0.7-0.8)$ for $\mathrm{Cd}_{1-x} \mathrm{Zn}_{x} \mathrm{Te}$ crystals is more suitable for operation at room temperature of high-resolution $\mathrm{X}$-ray and gamma-ray spectroscopy than $\mathrm{Cd}_{0.9} \mathrm{Zn}_{0.1} \mathrm{Te}$ [25].Therefore it is very important task to study $\mathrm{Cd}_{1-x} \mathrm{Zn}_{x}$ Te thick films with large $\mathrm{Zn}$ concentration. Earlier, different properties of thin and thick films have been studied in Refs. [10,26-28]. It was shown [26] that $\mathrm{Cd}_{1-x} \mathrm{Zn}_{x} \mathrm{Te}(x \leq 0.54)$ layers with typical thicknesses of $2-3 \mu \mathrm{m}$ deposited by molecular beam epitaxy on GaAs substrates observe narrow (PL) bands at higher energies which are attributed to bound exciton (BE) recombinations and the broader bands e-A, which arising from band-to-acceptor radiative transitions. The BE broadenings are attributed to statistical fluctuations in the concentrations of the cation atoms. XRD study of the thick polycrystalline $\mathrm{Cd}_{1-x} \mathrm{Zn}_{x} \mathrm{Te}$ films were done in Ref. [27] using the thermal evaporation method on carbon substrate, where the high signal-to-noise ratios of the X-ray detector have been obtained. A wide range of compositions of $\mathrm{Cd}_{1-x} \mathrm{Zn}_{x} \mathrm{Te}$ thick films on glass substrates were produced by metal-organic chemical vapor deposition [10], where it was shown that these films were composed of a single phase and were structurally stable. It was shown [10] that PL spectra in the range of the $\mathrm{Cd}_{1-x} \mathrm{Zn}_{x} \mathrm{Te}$ band gap present intense peaks, corresponding to $\mathrm{BE}$ and relatively wide bands due to defect-impurity recombination. However, identification of defect structure has not been attempted in these papers $[10,26]$. The crystalline quality of large grain size $\mathrm{Cd}_{1-x} \mathrm{Zn}_{x} \mathrm{Te}$ with high $\mathrm{Zn}$ concentrations $(0.45<x<0.85)$ grown by a zone melting process was investigated using their complex study [28]. It was found that the large Te precipitates are strongly reduced with the increase of $\mathrm{Zn}$ concentration.

In this work, we study the PL spectra of $\mathrm{Cd}_{1-x} \mathrm{Zn}_{x}$ Te thick films deposited on the Mo coated glass substrates by means of the CSVS technique. This let us determine the nature and the energy levels of point defects, their complexes and the residual impurities as well as the dislocations in the films. Furthermore we study the effect of $\mathrm{Zn}$ concentration on their defect's structure for $x \leq 0.67$. We have shown the presence of exciton acceptor complexes as well as the localized excitons in the PL spectra at large $\mathrm{Zn}$ concentrations. This let us determine the band gap energy of the films and study their inhomogeneity.

\section{Experimental details}

$\mathrm{Cd}_{1-\chi} \mathrm{Zn}_{x} \mathrm{Te}$ thick films were obtained by the close-spaced vacuum sublimation technique analogously as in Refs. [17,29] in the case of CdTe deposition. In order to obtain ternary $\mathrm{Cd}_{1-x} \mathrm{Zn}_{x} \mathrm{Te}$ compound films, two independent evaporators for CdTe and ZnTe materials were used. The stoichiometric CdTe and ZnTe powders were placed separately into different evaporators.

In order to obtain $\mathrm{Cd}_{1-x} \mathrm{Zn}_{x}$ Te films with different compositions, i.e. the mass ratio $\left(M_{R}\right)$ of CdTe to ZnTe powder was changed as 8, 4, 3 and 2 for S2, S3, S4 and S5 films samples, respectively. Besides thick films of binary compounds CdTe (S1) and ZnTe (S6) were obtained by the same method. It should be noted that the growth conditions of different samples were the same. The substrate temperature was $400^{\circ} \mathrm{C}$. The temperatures of the CdTe and ZnTe evaporators were $620^{\circ} \mathrm{C}$ and $700{ }^{\circ} \mathrm{C}$, respectively. The ultrasonically cleaned Mo coated glass slides were used as substrates.

Structural analysis was carried out using Rigaku Ultima + X-ray diffractometer with $\mathrm{K} \alpha \mathrm{Cu}$ radiation source, the scan step was 0.003 degrees 2-theta. Obtained X-ray patterns were used for phase analysis as well as for determination of the microstresses from the broadening of XRD peaks $[17,30]$. Namely, the calculation of stress values was performed using the equation $\varepsilon=\beta /(4 \tan \Theta)$, where $\beta$-instrumental broadening of the diffraction peak and $\Theta$ diffraction angle, i.e. analogously as that was done in Ref. [30]. The values of $\mathrm{Zn}$ concentration in $\mathrm{Cd}_{1-x} \mathrm{Zn}_{x} \mathrm{Te}$ thin films were determined by Vegard's law using lattice parameter calculated from position of ( $\left.\begin{array}{lll}1 & 1 & 1\end{array}\right)$ XRD line.

Surface morphology was investigated by FEI Nova NanoSEM 650 Schottky field emission scanning electron microscope (SEM). Average grain size $(d)$ in the layers was estimated by the Jeffries method [31]. Thickness of the films was measured by the SEM directly from the sample cross-section.

The PL measurements were carried out using a standard setup with a fully automated $1-\mathrm{m}$ focal length monochromator. A CW diode pumped solid state laser was used for excitation by the $457 \mathrm{~nm}(2.71 \mathrm{eV})$. The PL spectra were measured using a holographic grating $1200 \mathrm{~mm}^{-1}$ with the spectral dispersion of $0.8 \mathrm{~nm} / \mathrm{mm}$. The PL spectra were obtained by a thermoelectrically cooled, high efficiency extended-red multi-alkali cathode photomultiplier operating in the photon counting regime. The sample temperature was equal to $4.5 \mathrm{~K}$ using a helium optical cryostat.

\section{Results and discussion}

\subsection{Characterization of $\mathrm{Cd}_{1-x} \mathrm{Zn}_{x} \mathrm{Te}$ films}

The SEM images of the $\mathrm{Cd}_{1-x} \mathrm{Zn}_{x}$ Te samples are presented in Fig.1. It is found that the films obtained have a multi-grain structure with the grain size $d$ from $5.0 \mu \mathrm{m}$ to $8.0 \mu \mathrm{m}$. As can be seen from Fig. 1, there is a significant change in the surface morphology of different films that depends on mass ratio of CdTe to ZnTe powders. In particular, sample S2 includes quite uniformly distributed grains with the average size of $8 \mu \mathrm{m}$. In the case of sample S3 its surface is less uniform and the value of $d$ decreases to $5 \mu \mathrm{m}$. The value of $d$ for sample S4 is equal to $6.0 \mu \mathrm{m}$. At the same time, most grains include subgrains with size of about $1 \mu \mathrm{m}$. The surface of sample S5 consists of layered-like crystallites with irregular shapes and the $d$ value of about $7 \mu \mathrm{m}$. Such change of the surface could be explained by the strongly inhomogeneity of the crystal lattice as a result of large amount of $\mathrm{Zn}$ atoms in this film. This happens possibly due to more favorable growth condition for CdTe than ZnTe films, since the temperatures difference between evaporator and substrate $\Delta T=T_{e}-T_{s}$, is $220^{\circ} \mathrm{C}$ and $300^{\circ} \mathrm{C}$ for CdTe and $\mathrm{ZnTe}$, respectively. The thickness of the $\mathrm{Cd}_{1-x} \mathrm{Zn}_{x} \mathrm{Te}$ samples measured from the cross-section was about $30 \mu \mathrm{m}$.

The XRD patterns of the samples show the peaks of (1 111$)$, (2 00 0), (2 20 ), (3 11 1), (4 00 ), (3 31 1), (4 22 2) and (5 11 1) planes of the cubic zinc blende phase. It was found that the peaks on XRD patterns of the S2 to S5 samples are shifted toward higher degrees relatively to peak positions in CdTe because of increasing in $\mathrm{Zn}$ concentration. The value of $\mathrm{Zn}$ concentration determined from XRD data by Vegard's law [10] for S2 to S4 samples corresponds to $x=0.10,0.32$ and 0.44 , respectively. For these samples we observed sharp and symmetrical XRD diffraction peaks. This 

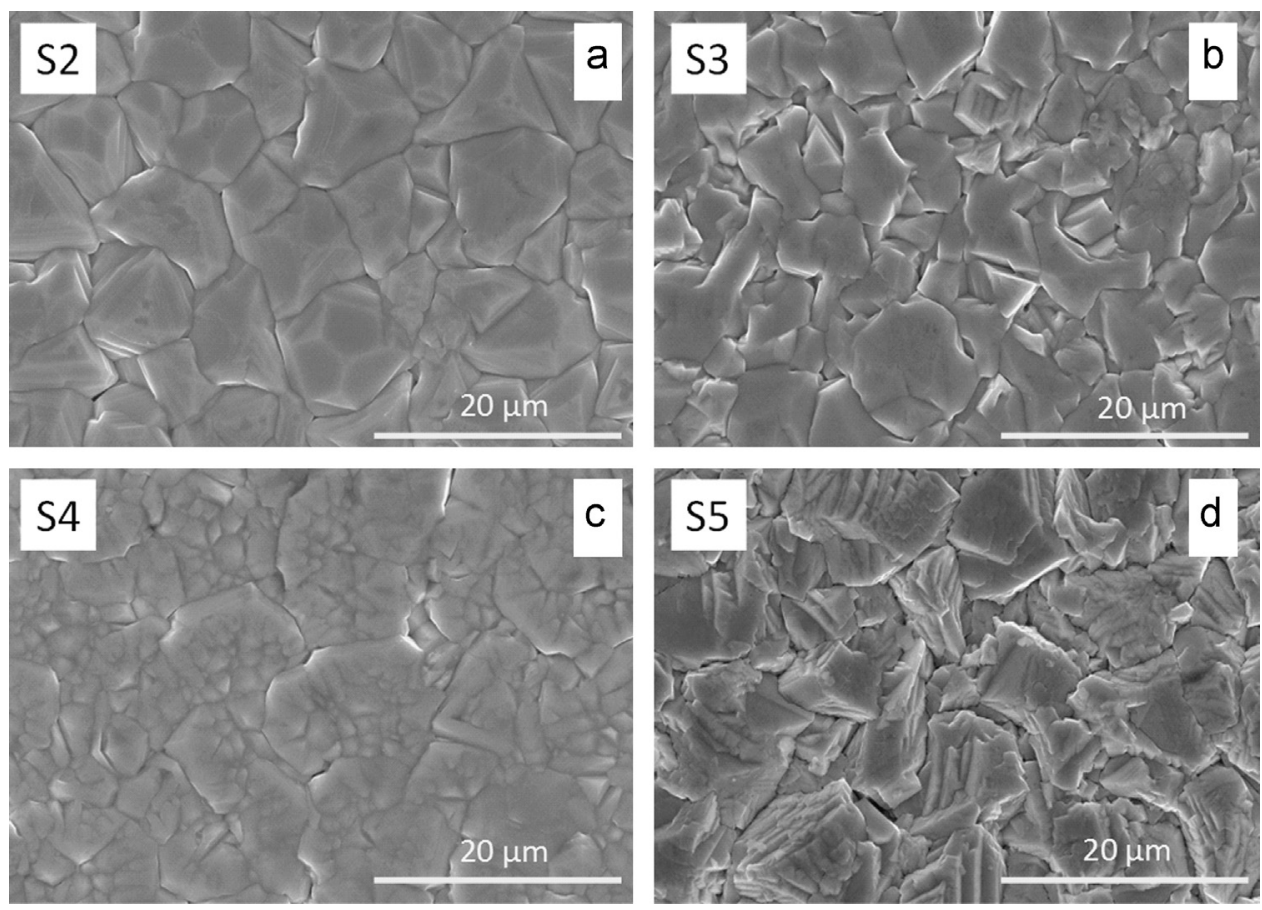

Fig. 1. SEM images of CZT samples surface: (a) S2, (b) S3, (c) S4, (d) S5.

indicates the uniformity of the $\mathrm{Zn}$ concentration in the films. In the case if the distribution of $\mathrm{Zn}$ concentration is nonuniform in thickness (i.e. the film includes a number layers with different $\mathrm{Zn}$ concentrations) the splitting of XRD peaks should be observed since the position of the peaks strongly depend on $\mathrm{Zn}$ concentration. This situation occurs for sample S5, where (1 111 1) XRD peak includes several components. So, for this sample the $\mathrm{Zn}$ concentration $(x=0.67)$ can be considered as an average value.

It should be noted that for sample $S 5$ the position of the diffraction peak of ( $\left.\begin{array}{lll}1 & 1 & 1\end{array}\right)$ plane corresponds to the concentration of $x=0.67$. At the same time, the shape of this peak is complex, indicating strong heterogeneity of component composition. It was shown that the microstress level in CdTe thick films increase from samples S2 to S5. In particular, for samples S2 to S5 the stress values correspond to $3.89 \times 10^{-3}, 5.54 \times 10^{-3}, 7.22 \times 10^{-3}$ and $13.3 \times 10^{-3}$, respectively. This indicates that the $\mathrm{Zn}$-poor films have lower growth stress than Zn-rich samples.

\subsection{Photoluminescence of $C d_{1-x} Z n_{x} T e$ films}

The low-temperature PL measurements provide powerful tools for the investigation of defect structure in different semiconductor materials [20,32,33]. This makes it possible to determine the nature and energy levels of both the intrinsic defects and the residual impurities as well as the relative concentration of dislocations in semiconductor materials [34,35]. It should also be noted that excitonic PL lines are very sensitive to various defects in semiconductors. The excitation energy of free excitons is slightly less than the energy of the unbound electron and hole, i.e. the band gap energy $E_{g}$ of semiconductors. Excitonic signatures in the low-temperature PL spectra of semiconductor compounds of high optical quality usually appear as the intense lines caused by the excitons bound to neutral (or ionized) donor and acceptor centers, i.e. the bound excitons (BE). In this case the energy levels of the $\mathrm{BE}$ move down from the free exciton level by the amount equal to their energy binding. This energy depends on the nature of the impurities or intrinsic defects. Therefore the energy position of the
$\mathrm{BE}$ let us determine the nature of defects participating in the formation of the excitonic complexes.

For semiconductor alloys, the crystal potential field fluctuations (CPFFs) caused by the random distribution of alloy components occur [36-38]. Excitonic states are localized at these fluctuations. This is due to the localization either as a whole exciton or as a hole with an electron bound to it by Coulomb interaction. The latter case may occur for CdTe crystal since the effective mass of the hole is 10 times higher than the effective mass of the electron. The lowenergy tails in the density of excitonic states arise [39,40]. At low temperatures localized excitons occupy the lowest energy states in these tails. When temperature is rising the localized excitons are excited to the higher energy states. It should be noted that the energy position of the localized excitons is shifted to the lowenergy region relative to the energy of free excitons by the amount corresponding to the energy about $1 / 2$ of the full width at halfmaximum (FWHM) of the localized exciton band. Taking into account the binding energy of free exciton for CdTe and ZnTe crystals, which corresponds to $\sim 10 \mathrm{meV}$, we can determine the band gap of the crystals (or films).

Thus, for semiconductor solid solutions (bulk or films), the excitons are localized both near point defects and at CPFFs, which reflects inhomogeneity of alloy's composition. Localized excitons occur in low-temperature PL spectra of semiconductor crystals and their manifestation is fairly well studied for bulk II-VI compounds. At the same time, PL exciton lines for thin and thick semiconductor films usually identified only as BE lines, caused by the emission of excitons bound with donor or acceptor centers. Thus, the localization of excitons associated with the presence of a strong heterogeneity in the distribution of $\mathrm{Cd}$ and $\mathrm{Zn}$ cations is not considered. Analysis of the PL spectra presented below takes into account the presence in $\mathrm{Cd}_{1-x} \mathrm{Zn}_{x} \mathrm{Te}$ as bound and localized excitons.

PL spectra of the films obtained at different temperatures are shown in Fig. 2 for the samples $S_{1}$ and $S_{2}$. In this case sample $S_{1}$ corresponds to thick CdTe film obtained by CSVS method under growth conditions similar to $\mathrm{Cd}_{1-x} \mathrm{Zn}_{x} \mathrm{Te}$ films. Analysis of the observed PL spectrum reveals an intense excitonic emission at 

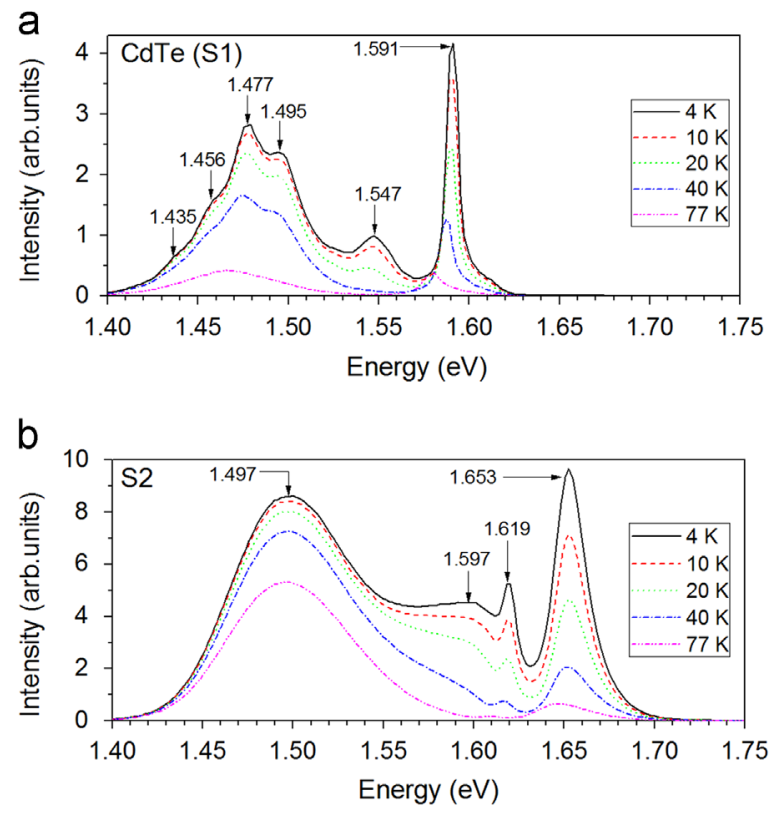

Fig. 2. PL spectra of the samples: (a) CdTe (S1), (b) S2.

$E=1.591 \mathrm{eV}$, which is caused by the recombination of excitons bound on a neutral acceptor ( $\mathrm{A}^{\circ} \mathrm{X}$-line) with the participation of cadmium vacancy $\left(\mathrm{V}_{C d}\right)$ [41]. The presence of this PL line indicates p-type conductivity of CdTe sample. The energies of this line are at $1.591 \mathrm{eV}, 1.591 \mathrm{eV}, 1.589 \mathrm{eV}$ and $1.587 \mathrm{eV}$ for different temperatures $(4,10,20$ and $40 \mathrm{~K}$, respectively) and reflect the temperature dependence of $E_{g}$. As mentioned above, the energy of band gap corresponds to the energy position of $\mathrm{A}^{\circ} \mathrm{X}$-line $\left(E_{A}^{e x}\right)$ plus the binding energy of this BE, which for CdTe is equal to about $5 \mathrm{meV}$, as well as the binding energy of free exciton $(10 \mathrm{meV})$, i.e. $E_{g}=E_{A}^{e x}+15 \mathrm{meV}$ [17]. Thus the energy position of free exciton in CdTe film (S1 sample) corresponds to $1.596 \mathrm{eV}$ and the band gap is $1.606 \mathrm{eV}$. These energy values coincide with the corresponding energy for bulk CdTe crystal. The presence of intense exciton line indicates a fairly good optical quality of the investigated semiconductor films [19]. It should be noted that the shape of $A^{\circ} X$-line is practically symmetric. However its high-energy shoulder is slightly tightened as a result small intensity emission of free excitons ( $\mathrm{X}$-line) and excitons bound to neutral donor ( $\mathrm{D}^{\circ} \mathrm{X}$-line).

It should be noted that the nature of $\mathrm{A}^{\circ} \mathrm{X}$-line may be supported by the analysis of PL spectrum in the long-wavelength spectral range where the PL band at $1.547 \mathrm{eV}$ is associated with the recombination of free electrons and acceptor centers ((e,A)-transition) $[1,16]$. Since this line is broadened, we may assume that other $\mathrm{A}^{\circ} \mathrm{X}$-exciton complexes also participate in the exciton emission but their intensity is smaller than the above mentioned $A$ ${ }^{\circ} \mathrm{X}$-line at $1.591 \mathrm{eV}$. Thus, knowing the energy of this PL line and the value of $E_{g}(4.5 \mathrm{~K})=1.606 \mathrm{eV}$ for $\mathrm{CdTe}$ film, we can determine the energy of the acceptor level. According to Refs. [42,43] the energy of (e,A)-transition can be presented by the following equation:

$E(\mathrm{e}, \mathrm{A})=E_{\mathrm{g}}-E_{A}+\S k T$,

where $E_{A}$ is the acceptor energy.

Using Eq. (1) we obtain the energy of the acceptor center associated with (e,A)-transition, namely $E_{A}=59 \mathrm{meV}$. This value is close to the acceptor energy of $\mathrm{Li}$ or $\mathrm{Na}(58.0 \mathrm{meV}$ and $58.7 \mathrm{meV}$, respectively) [44]. The energy of the both exciton acceptor complexes is about $1.589 \mathrm{eV}$ [44]. This indicates that $\mathrm{A}^{\circ} \mathrm{X}$-line is complex and consists of the overlaping emission of several exciton acceptor complexes. However, the intensity of this line is mainly determined by the emission from the complex involving cadmium vacancy. The presence of small asymmetry in $A^{\circ} \mathrm{X}$-line (the ratio of high-energy part of FWHM to low-energy part of FWHM (B) is about of 0.9) may be caused by the participation of other exciton acceptor complexes. In this spectral range, the emission can also appear due to the recombination of donor-acceptor pairs. However, it is obvious that intensity of such emission is very small, since the structure on low-energy wing of this band associated with (e,A)-transition does not appear in the PL spectrum. It indicates that the donor concentration in the investigated CdTe thick film is small.

As can be seen from Fig. 2a, a group relatively broad PL bands in the spectral range $1.40-.57 \mathrm{eV}$ is observed. The band at $1.495 \mathrm{eV}$ can be caused by the recombination of donor-acceptor pairs (DAP) with the participation of shallow donor and the acceptor complex $\left[\mathrm{V}_{C d}-\mathrm{D}\right]$, where $\mathrm{D}$ is residual donor (atoms of III or VII group metals) and $\mathrm{V}_{C d}$ is a cadmium vacancy. The difference between the value of $E_{g}=1.606 \mathrm{eV}(T=4.5 \mathrm{~K})$ for $\mathrm{CdTe}$ and the peak position of the band is equal to $111 \mathrm{meV}$. The energy of the shallow $\mathrm{Cl}$ donor in CdTe is equal to $14 \mathrm{meV}$ [45]. In this case the energy of the emitted light, associated with zero-phonon $\mathrm{I}_{\mathrm{DAP}}$ - line, is given by the following equation $[46,47]$ :

$E=E_{g}-\left(E_{D}+E_{A}\right)+e^{2} / \varepsilon_{0} \varepsilon R$

where $E_{g}$ is the band gap energy, $E_{D}$ and $E_{A}$ are the ionization energies of donor and acceptor centers, respectively, $e^{2} / \varepsilon_{0} \varepsilon R$ is the Coulomb energy of the two ionized impurity centers (donor and acceptor pairs) separated by distance $R, \varepsilon$ is the dielectric constant of the investigated material, $e$ is the elementary charge. The Coulomb energy of donor-acceptor pairs in CdTe is equal to $\sim 20 \mathrm{meV}$ for residual impurities. Therefore, it is expected that the energy of acceptor center is about $117 \mathrm{meV}$. It should be noted that this energy is close to the energy of $\mathrm{Ag}$ acceptor center (107 meV) [44]. At the same time the acceptor energy is almost coincides with the energy of an $\mathrm{A}$ center where a $\mathrm{Cd}$ vacancy paired with a $\mathrm{Cl}$ donor, namely, $\left(\mathrm{V}_{C d}-\mathrm{Cl}_{T e}\right)$ acceptor center $(120 \mathrm{meV})[48,49]$. Usually, emission of DAPs is accompanied by the presence of additional long-wavelength PL bands caused by the optical transitions with the participation of LO-phonons with the energy of $21 \mathrm{meV}$. In this case it is difficult to see such bands since other intense PL band at $1.477 \mathrm{eV}$ and its $1 \mathrm{LO}-$ and 2LO-phonons replicas are observed at 1.456 and $1.435 \mathrm{eV}$, respectively. For the samples which have high density of dislocations and are strongly inhomogeneous it is difficult to observe these LO-phonon replicas. Therefore, we assume that CdTe film have very good optical quality.

The PL band at $1.477 \mathrm{eV}$ is due to Y center which corresponds to excitons bound on Te glide dislocations [49-52]. The nature of this band is supported by the results for CdTe crystal sample obtained from the bottom of the crystal bar where $Y$ band strongly increases [50]. Thus, the intensity of the Y band may be used as an indicator of the dislocations in CdTe.

The PL spectrum for $\mathrm{Cd}_{1-x} \mathrm{Zn}_{x} \mathrm{Te}$ (sample S2) is shown in Fig. $2 \mathrm{~b}$ where the intense exciton emission at $1.653 \mathrm{eV}$ is caused by the recombination of excitons bound on a neutral acceptor ( $\mathrm{A}^{\circ} \mathrm{X}$-line). The energy position of this line is the same for the temperature range $4.5-40 \mathrm{~K}$ unlike that for sample $\mathrm{S} 1$ where its temperature dependence is similarly to $E_{g}$. This value is equal to $1.668 \mathrm{eV}$ at $T=4.5 \mathrm{~K}$. It should be noted that this line has high-energy asymmetry. The value of $B$ is changed from 1.1 to 1.40 for temperatures from $4.5 \mathrm{~K}$ to $40 \mathrm{~K}$, respectively. We assume that such behavior of this exciton line is caused by some overlapping of $\mathrm{A}^{\circ} \mathrm{X}$-line with the lines of localized excitons appearing on its high-energy wing. In this case $\mathrm{A}^{\circ} \mathrm{X}$-line is the crucial. The temperature dependence of the $B$ value reflects the increase of the contribution of the localized excitons when the temperature of sample S2 increases. The 
temperature dependence of the $B$ value reflects increase of the contribution of the localized excitons when the temperature of sample S2 increases. The FWHM of this line strongly increases in comparison with that for S1 sample (23 and $7 \mathrm{meV}$, respectively) that indicates inhomogeneity of sample S2 as a result of solid solution formation and the presence of other defects. In this case, CPFFs, caused by the random distribution of alloy components, leads to the appearance of the localized excitons. Other line at $1.619 \mathrm{eV}$ is observed which may be caused by the recombination of DAP or (e,A)-transitions. As was shown above the value of $E_{g}$ for sample S2 is equal to $1.668 \mathrm{eV}$. So the difference between these energies is $49 \mathrm{meV}$ which corresponds to the most shallow acceptor level in CdTe associated with the cadmium vacancy. In the case of DAP transitions the energy of donor level is equal to 14 mev. This means that acceptor center should have the ionization energy equal to $35 \mathrm{meV}$. However, CdTe do not have the acceptor with such energy. Therefore we assume that the line at $1.619 \mathrm{eV}$ is caused by the (e,A)-transitions. In this case the acceptor energy is equal to $49 \mathrm{meV}$ that corresponds to the cadmium vacancy acceptor. The FWHM of this line is smaller than for sample S1 where the broadening may be caused by the (e,A)-transitions with the participation of the acceptors with different ionization energies. Besides $\mathrm{V}_{C d}$-acceptor here may be different acceptors caused by the presence of residual impurities such as $\mathrm{Li}, \mathrm{Na}, \mathrm{N}$ and $P$ with the energies of 58.0, 58.7, 56.0 and $68.2 \mathrm{meV}$ [44]. The contribution of these acceptors is determined by their concentration in the investigated samples. Thus, we assume that the concentration of above mentioned residual impurities in sample S2 is considerably smaller than that for sample $\mathrm{S} 1$. As can be seen from Fig. 2b 1LO-phonon replica of (e,A)-transition is observed at $1.597 \mathrm{eV}$. On the other hand, this energy may be caused by DAP transitions with the participation of shallow donor centers, which have ionization energy of $20 \mathrm{meV}$, and also can be caused by $\mathrm{Al}$ residual impurity atoms situated at $\mathrm{Cd}$ sites [53]. However, in that case we would have observed relative increasing of (e,A)-transition intensity with temperature raising [19] in the PL spectrum. Other very broad PL band at $1.497 \mathrm{eV}$ corresponds to so-called D PL band, caused by the presence of point and extended defects of dislocation type as well as microstrains in the films [50,54].

In Fig. 3a the PL spectrum for sample S3 is shown. Similar to the previous films the most intensive line is the exciton line at
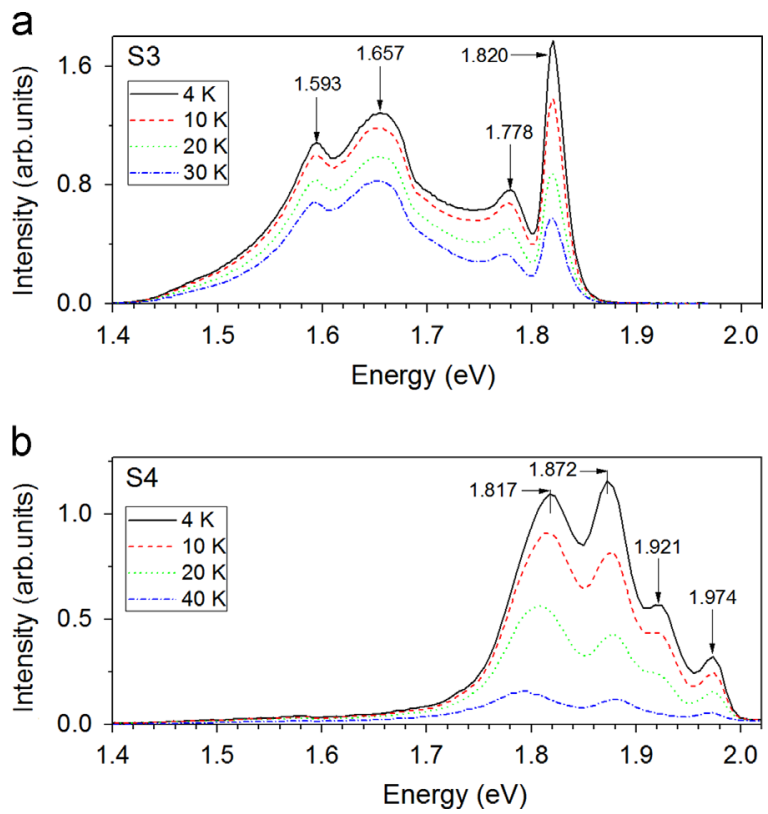

Fig. 3. PL spectra of the samples: (a) S3, (b) S4.
$1.820 \mathrm{eV}$. It should be noted that its FWHM is equals to $24 \mathrm{meV}$, i.e. practically coincides with the similar value for sample S2 (23 meV), although the zinc concentration for sample S3 is significantly higher. It is obvious that the rising of $\mathrm{Zn}$ concentration above $x=0.10$ leads to the increase of the contribution of the localized excitons which becomes dominant in the excitonic spectral region. In our opinion, this exciton line, which is practically symmetric, is mainly caused by the recombination of the localized excitons. The energy of this line is temperature independent at $T=4.5-40 \mathrm{~K}$. At the same time, the band gap of CdTe decreases in this temperature region for about $4 \mathrm{meV}$. This is due to the emission of localized excitons from their excited states when temperature increases. Thus, for sample S3 the emission of the localized excitons is dominant in the PL spectrum. Taking into account the energy value of $1 / 2$ FWHM, which is equal of $12 \mathrm{meV}$ for the localized excitons we assume that the band gap for sample S3 corresponds to $1.842 \mathrm{eV}$. The PL spectrum also includes three broad bands at $1.778,1.657$ and $1.593 \mathrm{eV}$. First band may be caused by $(\mathrm{e}, \mathrm{A})$-transition, where the acceptor energy is equal to $64 \mathrm{meV}$, i.e. is close to the ionization energy of residual impurities $(\mathrm{Li}, \mathrm{Na}$ and P) [44]. On the other hand, this band may be the result of DAP emission with the participation of donor and acceptor centers, which have the ionization energies $14 \mathrm{meV}$ and $50 \mathrm{meV}$, respectively. In this case, the acceptor center is caused by the presence of cadmium vacancy. It is obvious that the PL bands at 1.657 and $1.593 \mathrm{eV}$ correspond to the emission of DAP with the participation of A-centers and D band associated with the presence of dislocations.

As can be seen from Fig. 3b, the PL spectrum of sample S4 is shifted to the high-energy region in comparison with samples S3. Similar to sample S3, a high-energy line at $1.974 \mathrm{eV}$ corresponds to the recombination of localized excitons and its energy is temperature independent. Decreasing $\left(\mathrm{A}^{\circ} \mathrm{X}\right)$-line intensity may be caused by increasing microstrains and decreasing coherent scattering domains for sample S4. Taking into account the energy value of $1 / 2$ FWHM for this exciton line $(12 \mathrm{meV})$ we can determine the value $E_{g}$, which for this sample is equal to $1.996 \mathrm{eV}$. The other PL band at $1.921 \mathrm{eV}$ may be caused by the (e,A)-transition with the participation of the acceptor center, which have the energy of $75 \mathrm{meV}$ or more likely to the recombination of DAPs with the participation of the donor and acceptor centers with the energies of $14 \mathrm{meV}$ and $61 \mathrm{meV}$. The last energy is close to the energy of the acceptor centers caused by the presence of residual impurities such as Li or Na. The broad PL bands at 1.872 and $1.817 \mathrm{eV}$ correspond to the DAP recombination and the emission of Y-band, respectively. Therefore, it is expected that the energy of acceptor center is about $110 \mathrm{meV}$, which is close to the energy of Ag acceptor center (107 meV) [44].

In Fig. 4a PL spectrum for sample S5, containing the highest concentration of zinc atoms, is shown. The distinction of this spectrum is the presence of emission in the spectral region which is very close to CdTe film (sample S1). Particularly, the line at $1.585 \mathrm{eV}$ is very close to the energy position of $\left(\mathrm{A}^{\circ} \mathrm{X}\right)$-line. Other line at $1.546 \mathrm{eV}$ may be caused by $(\mathrm{e}, \mathrm{A})$-transition. In this case the acceptor energy is equal to about $60 \mathrm{meV}$ and is practically coincides with the energy of $\mathrm{Li}$ and $\mathrm{Na}$ acceptors. The emission at $1.495 \mathrm{eV}$ also coincides with the recombination of DAP of the acceptor, which has the energy $111 \mathrm{meV}$ similar to CdTe film sample S1. The broadening of these lines is caused by the presence of high concentration of microstrains in the investigated sample. This is supported by the very broad band at $1.796 \mathrm{eV}$ in PL spectrum a, which is most likely related to the emission with the participation of various extended defects, especially dislocations. In the high-energy spectral range the blurred PL band is observed at the energy of about $2.100 \mathrm{eV}$. This band can be determined as strongly broadened line of localized excitons. In this case, 1/2 

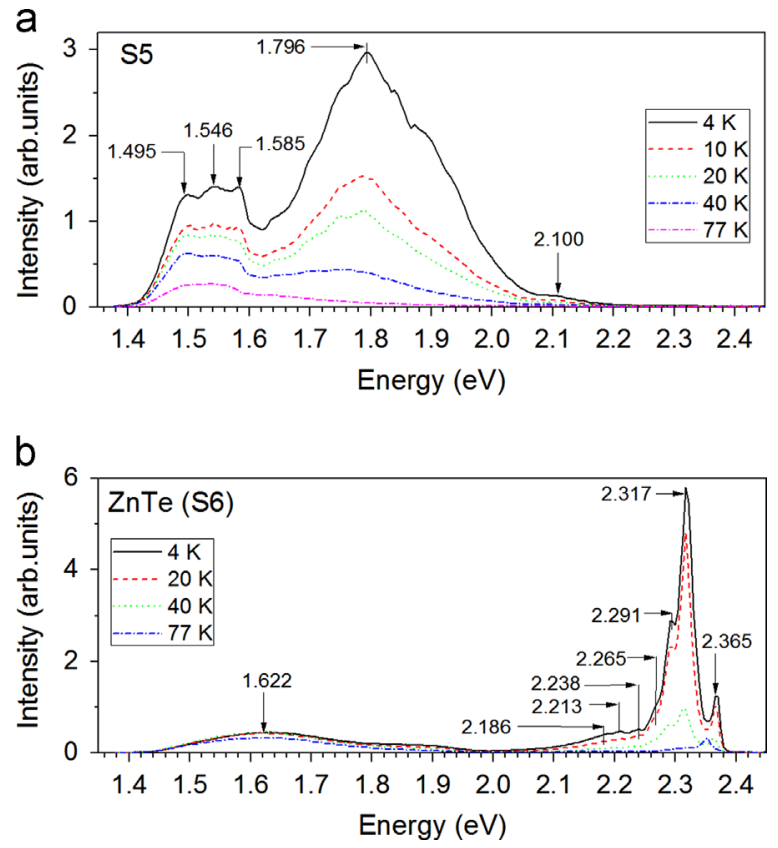

Fig. 4. PL spectra of the samples: (a) S5, (b) S6.

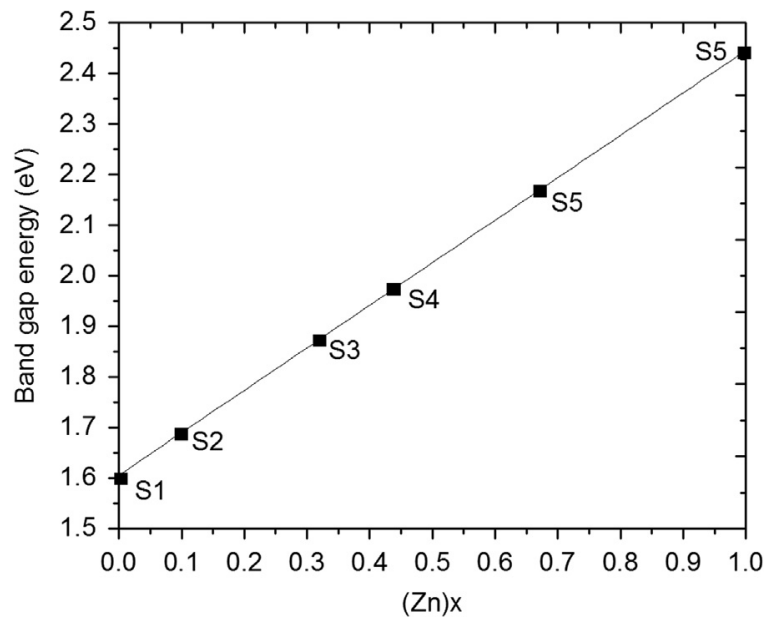

Fig. 5. $E_{g}(x)$ dependence for $\mathrm{Cd}_{1-x} \mathrm{Zn}_{x}$ Te thick films.

FWHM for this exciton line is approximately $40 \mathrm{meV}$. Thus, the band gap for $\mathrm{Zn}$-rich region film of sample S5 can be equal to about $2.150 \mathrm{eV}$. In Fig. 5 the concentration dependence of the band gap of the investigated $\mathrm{Cd}_{1-x} \mathrm{Zn}_{x}$ Te thick films is presented. As can be seen from this figure, the band gap of these films is approximated by linear dependence, which can be described by the following equation:

$E_{g}(x)=1.606 \mathrm{eV}+0.79 x$

In Fig. 4b the PL spectrum for sample S6 (ZnTe film) is shown. The line with highest energy at $2.365 \mathrm{eV}$ corresponds to $\mathrm{A}^{\circ} \mathrm{X}$-line and is caused by the zinc vacancy acceptor exciton complex [18]. According to Ref. [18] the energy position of free exciton in ZnTe films corresponds to $2.384 \mathrm{eV}$. Taking into account the binding energy of free exciton ( $11 \mathrm{meV}$ ) we can determine the band gap of ZnTe film, which is $2.395 \mathrm{eV}$. The most intensive line at $2.317 \mathrm{eV}$ is caused by the emission of DAP's and is zero-phonon line. In this case, $\mathrm{Cl}_{T e}$ donor with the ionization energy of $22 \mathrm{meV}$ and $\mathrm{V}_{Z n}$ acceptor with the ionization energy of $56 \mathrm{meV}$ [18] participate respectively. Other lines observed on the long-wavelength of this line at $2.291 \mathrm{eV}, 2.265 \mathrm{eV}, 2.238 \mathrm{eV}, 2.213 \mathrm{eV}$ and $2.186 \mathrm{eV}$ are due to the 1LO-, 2LO-, 3LO-, 4LO- and 5LO-phonon replicas of the line at $2.317 \mathrm{eV}$. The broad band at $1.622 \mathrm{eV}$ is caused by the emission with participation of deep impurity level.

In summary, we studied the low-temperature PL spectra of $\mathrm{Cd}_{1-x} \mathrm{Zn}_{x} \mathrm{Te}$ thick films deposited on transparent glass substrate at different $\mathrm{Zn}$ concentration by means of close-spaced vacuum sublimation technique. Structural analysis showed that the films have only cubic zinc blende phase. It has been found that the films have a dispersed structure with the average grain size from $5.0 \mu \mathrm{m}$ to $8.0 \mu \mathrm{m}$. Thickness of the films was about of $30 \mu \mathrm{m}$. It was shown that the PL spectrum of the film for $x=0.10$ exhibits an intense acceptor bound exciton $\mathrm{A}^{\circ} \mathrm{X}$-line, which is characteristic for $\mathrm{Cd}_{1-x} \mathrm{Zn}_{x}$ Te single crystals of $\mathrm{p}$-type, as well as other bands caused by donor-acceptor emission with the participation of $\mathrm{Na}, \mathrm{Li}, \mathrm{P}$ and $\mathrm{Ag}$ residual impurities. Other very broad PL bands were observed, which correspond to so-called $\mathrm{D}$ and $\mathrm{Y}$ bands caused by the point and extended defects as well as microstrains in the films. It should be noted that the $\mathrm{A}^{\circ} \mathrm{X}$-line for $x=0.10$ shows a short-wavelength asymmetry under elevated temperature that is due to the occurrence of the lines of localized excitons. For the films with higher $\mathrm{Zn}$ concentration the line of localized excitons is dominant in the region of exciton emission. The presence of an intense excitonic emission for samples S2 $(x=0.10)$ and S3 $(x=0.32)$ indicates the fairly good optical quality of these films. For the $\mathrm{Cd}_{1-x} \mathrm{Zn}_{x} \mathrm{Te}$ film with $x=0.67$ strongly inhomogeneous PL spectrum structure was observed. In this case the PL spectrum shows the emission, which is characteristic for almost pure CdTe crystal and an intense D PL band, which is caused by strongly inhomogeneous $\mathrm{Zn}$ distribution near the film surface. Taking into account the energy position of exciton lines the concentration dependence of the band gap for the investigated $\mathrm{Cd}_{1-x} \mathrm{Zn}_{x}$ Te thick films was presented. The correlation between the broadening of exciton lines and the value of microstress in $\mathrm{Cd}_{1-x} \mathrm{Zn}_{x}$ Te thick films was also determined.

Our findings provide a way to obtain thick polycrystalline $\mathrm{Cd}_{1-x} \mathrm{Zn}_{x} \mathrm{Te}$ films deposited on a Mo coated glass substrate by means of CSVS with large $\mathrm{Zn}$ concentration suitable for application in X-ray detectors. In future, more detailed study of the structural properties of $\mathrm{Cd}_{1-x} \mathrm{Zn}_{x}$ Te thick films will be carried out.

\section{Acknowledgments}

This work was supported by Grant State Fund for Fundamental Research (project N GP / F61 / 087) and by the Ministry of Education and Science of Ukraine (Grant no. 0110U001151) by the National Academy of Sciences of Ukraine (Grants nos. BC-157-15 and B-146-15).

\section{References}

[1] R. Triboulet P. Siffert, CdTe and Related Compounds; Physics, Defects, Heteroand Nano-structures, Crystal Growth, Surfaces and Applications, Elsevier, Amsterdam, 2009.

[2] A. Owens, Compound Semiconductor Radiation Detectors, CRC Press, Boca Raton, 2012.

[3] S. del Sordo, L. Abbene, E. Caroli, A.M. Mancini, A. Zappettini, P. Ubertini, Sensors 9 (2009) 3491.

[4] M. Fiederle, T. Feltgen, J. Meinhardt, M. Rogalla, K.W. Benz, J. Cryst. Growth 197 (1999) 635.

[5] F.P. Doty, J. Vac. Sci. Technol. B Microelectron. Nanometer Struct. 10 (1992) 1418.

[6] F. Tao, Z. Gangqiang, Y. Jian, L. Jiong, J. Zheng, X. Lingyan, et al., J. Phys. Conf. Ser. 430 (2013).

[7] P.J. Sellin, Thick film compound semiconductors for X-ray imaging applications, Nucl. Instruments Methods Phys. Res. Sect. A Accel. Spectrometers, Detect. Assoc. Equip.

[8] S. Kang, B. Jung, S. Noh, C. Cho, I. Yoon, J. Park, J. Instrum. 7 (2012).

[9] M. Niraula K. Yasuda S. Namba T. Kondo S. Muramatsu Y. Wajima, et al., MOVPE growth of thick single crystal CdZnTe epitaxial layers on Si 
substrates for nuclear radiation detector development, in: Proceedings of IEEE Nuclear Science Symposium and Medical Imaging Conferenc Record.

[10] S. Stolyarova, F. Edelman, a Chack, a Berner, P. Werner, N. Zakharov, et al., J. Phys. D Appl. Phys. 41 (2008) 065402.

[11] A. Aydinli, A. Compaan, G. Contreras-Puente, A. Mason, Solid State Commun. 80 (1991) 465.

[12] J.H. Won, K.H. Kim, J.H. Suh, S.H. Cho, P.K. Cho, J.K. Hong, et al., Instrum. Methods Phys. Res. Sect. A: Accel. Spectrom. Detect. Assoc. Equip. 591 (2008).

[13] K. Prabakar, S. Venkatachalam, Y.L. Jeyachandran, S.K. Narayandass, D. Mangalaraj, Mater. Sci. Eng. B Solid-State Mater. Adv. Technol. 107 (2004) 99.

[14] K.R. Murali, Sol. Energy. 82 (2008) 220-225.

[15] H. Zhou, D. Zeng, S. Pan, Instrum. Methods Phys. Res. Sect. A: Accel. Spectrom. Detect. Assoc. Equip. 698 (2013) 81.

[16] J. Takahashi, K. Mochizuki, K. Hitomi, T. Shoji, J. Cryst. Growth 269 (2004) 419.

[17] V. Kosyak, A. Opanasyuk, P.M. Bukivskij, Y.P. Gnatenko, J. Cryst. Growth 312 (2010) 1726.

[18] Y.P. Gnatenko, P.M. Bukivskij, AS. Opanasyuk, D.I. Kurbatov, M.M. Kolesnyk, V. V. Kosyak, et al., J. Lumin. 132 (2012) 2885.

[19] Y.P. Gnatenko, P.M. Bukivskij, I.O. Faryna, A.S. Opanasyuk, M.M. Ivashchenko, J. Lumin. 146 (2014) 174.

[20] Y.P. Gnatenko, A.S. Opanasyuk, M.M. Ivashchenko, P.M. Bukivskij, I.O. Faryna, Mater. Sci. Semicond. Process. 26 (2014) 663.

[21] Y.P. Gnatenko, P.M. Bukivskij, Y.P. Piryatinski, I.O. Faryna, O.A. Shigiltchoff, R. V. Gamernyk, Appl. Phys. Lett. 95 (2009) 112109.

[22] Y.P. Gnatenko, Y.P. Piryatinski, R.V. Gamernyk, I.O. Faryna, P.M. Bukivskij, S. Y. Paranchych, et al., Proc. SPIE -Int. Soc. Opt. Eng. 5209 (2003) 156.

[23] J. Zazvorka, J. Franc, P. Hlidek, R. Grill, J. Lumin. 143 (2013) 382.

[24] I. Nasieka, N. Kovalenko, V. Kutniy, A. Rybka, D. Nakonechnyj, S. Sulima, et al., Sens. Actuators A Phys. 203 (2013) 176.

[25] J.E. Toney, T.E. Schlesinger, R.B. James, Instrum. Methods Phys. Res. Sect. A: Accel. Spectrom. Detect. Assoc. Equip. 428 (1999) 14.

[26] D.J. Olego, J.P. Faurie, S. Sivananthan, P.M. Raccah, Appl. Phys. Lett. 47 (1985) 1172.

[27] S. Yuk, S.W. Park, Y. Yi, Polycrystalline CdZnTe thick films for low energy X-ray: system evaluation, in: Proceedings of Annual International Conference of IEEE Engineering in Medicine and Biology, 2006, p. 1990-1993.

[28] J. Pérez Bueno, M. Rodríguez, O. Zelaya-Angel, R. Baquero, J. GonzalezHernández, L. Baños, et al., J. Cryst. Growth 209 (2000) 701.

[29] D.I. Kurbatov, V.V. Kosyak, M.M. Kolesnyk, A.S. Opanasyuk, S.N. Danilchenko, Y. P. Gnatenko, Mater. Chem. Phys. 138 (2013) 731.

[30] B. Venugopal, B. Nandan, A. Ayyachamy, V. Balaji, S. Amirthapandian, B. K. Panigrahi, T. Paramasivam, RSC Adv. 4 (2014) 6141.
[31] G.F. Vander Voort, Metallography, Principles and Practice, ASM International, Materials Park, Ohio, 1984

[32] J.A. Garcia, A. Remon, V. Munoz, R. Triboulet, J. Mater. Res. 15 (2000) 1612.

[33] Y.P. Gnatenko, O.A. Shigil'chev, E. Rutkovskii, G. Contreras-Puente M. Cardenas-Garcia, Phys. Solid State 40 (1998) 564.

[34] M.S. Furyer, P.A. Skubenko, P.M. Bukivskij, L.M. Tarakhan, E.D. Chesnokov, I. G. Vertegel, et al., J. Appl. Phys. 108 (2010) 103711.

[35] Y.P. Gnatenko, M.S. Furyer, A.P. Bukivskii, L.M. Tarakhan, R.V. Gamernyk, J. Lumin. 160 (2015) 258.

[36] D. Ouadjaout, Y. Marfaing, Phys. Rev. B 41 (1990) 12096.

[37] L.G. Suslina, D.L. Fedorov, A.G. Areshkin, V.G. Melekhin, Solid State Commun. 55 (1985) 345

[38] S.D. Baranowskii, A.L. Efros, Fiz. Tekh. Poluprovodn. 12 (1978) 2233.

[39] E. Cohen, M.D. Sturge, Phys. Rev. B 25 (1982) 3828.

[40] M.A. Kanehisa, R.J. Elliott, Phys. Rev. B 35 (1987) 2228.

[41] J.M. Figueroa, F. Sanchez-Sinencio, J.G. Mendoza-Alvarez, O. Zelaya, C. Vázquez-López, J.S. Helman, J. Appl. Phys. 60 (1986) 452.

[42] D.M. Eagles, J. Phys. Chem. Solids 16 (1960) 76.

[43] V. Consonni, Optical properties of CdTe, in: R. Triboulet, P. Siffert (Eds.), CdTe and Related Compounds: Physics, Defects, Hetero- Nano-Structures, Crystal Growth, Surfaces Appllications Part: Physics, CdTe-Based Nanostructures, CdTe-Based Semimagnetic Semiconductors Defects, Elsevier, Amsterdam, 2010, pp. 59-84.

[44] E. Molva, J.L. Pautrat, K. Saminadayar, G. Milchberg, N. Magnea, Phys. Rev. B 30 (1984) 3344.

[45] J.M. Francou, K. Saminadayar, J.L. Pautrat, Phys. Rev. B 41 (1990) 12035.

[46] R. Tenne, H. Mariette, C. Levy-Clement, R. Jager-Waldau, Phys. Rev. B 36 (1987) 1204.

[47] Y. Kokubun, H. Watanabe, M. Wada, Jpn. J. Appl. Phys. 13 (1974) 1393.

[48] M. Soltani, M. Certier, R. Evrard, E. Kartheuser, J. Appl. Phys. 78 (1995) 5626.

[49] A. Castaldini, A. Cavallini, B. Fraboni, P. Fernandez, J. Piqueras, J. Appl. Phys. 83 (1998) 2121.

[50] S. Seto, A. Tanaka, F. Takeda, K. Matsuura, J. Cryst. Growth. 138 (1994) 346.

[51] S. Hildebrandt, H. Uniewski, J. Schreiber, H.S. Leipner, J. Phys. III 7 (1997) 1505.

[52] V. Babentsov, J. Franc, P. Höschl, M. Fiederle, K.W. Benz, N.V. Sochinskii, et al, Cryst. Res. Technol. 44 (2009) 1054.

[53] S.-H. Wei, S.B. Zhang, Phys. Rev. B. 66 (2002) 155211.

[54] J. Aguilar Hernandez, M. Cardenas Garcia, G. Contreras Puente, J. Vidallarramendi, Mater. Sci. Eng. B 102 (2003) 203. 